\title{
Cystic brain metastasis of non-small-cell lung cancer successfully controlled with Ommaya reservoir placement
}

\author{
Gouji Toyokawa $\cdot$ Ryo Toyozawa $\cdot$ Eiko Inamasu $\cdot$ Miyako Kojo $\cdot$ \\ Yosuke Morodomi - Yoshimasa Shiraishi - Tomoyoshi Takenaka • \\ Fumihiko Hirai • Masafumi Yamaguchi · Takashi Seto • \\ Mitsuhiro Takenoyama $\cdot$ Yukito Ichinose
}

Received: 7 August 2012/ Accepted: 23 October 2012/Published online: 21 November 2012

(C) The Japan Society of Clinical Oncology 2012

\begin{abstract}
A 68-year-old male presented with hoarseness and anarthria. Computed tomography showed an irregular nodular shadow in the upper lobe of the left lung with swollen multiple lymph nodes. Magnetic resonance imaging revealed a large cystic mass in the left hemisphere of the brain and multiple brain metastases in the bilateral hemispheres. A direct biopsy with bronchoscopy of the pulmonary nodule revealed the tumor to be an adenocarcinoma clinically diagnosed as stage IV. Since the largest brain metastasis continued to grow despite the administration of whole brain irradiation, insertion of an Ommaya reservoir in the cystic lesion was performed. This resulted in a reduction of the size of the brain tumor, and the patient's neurological symptoms improved. After the Ommaya reservoir was placed, stereotactic radiosurgery was performed on the largest lesion. The patient is doing well at 6 months after the Ommaya reservoir was inserted and is currently undergoing chemotherapy. In conclusion, the placement of an Ommaya reservoir may therefore be a potentially useful therapeutic procedure to improve the neurological symptoms and performance status in nonsmall-cell lung cancer patients with cystic brain metastasis, thereby allowing further neurosurgical therapy and chemotherapy.
\end{abstract}

Keywords Non-small-cell lung cancer - Cystic brain metastasis · Ommaya reservoir

G. Toyokawa $(\bowtie) \cdot$ R. Toyozawa $\cdot$ E. Inamasu - M. Kojo · Y. Morodomi - Y. Shiraishi - T. Takenaka - F. Hirai · M. Yamaguchi - T. Seto - M. Takenoyama - Y. Ichinose Department of Thoracic Oncology, National Kyushu Cancer Center, 3-1-1 Notame, Minami-ku, Fukuoka 811-1395, Japan e-mail: gouji104kawa@gmail.com

\section{Introduction}

An Ommaya reservoir is a device placed under the skin of the head with the tip of the catheter positioned into the ventricles or within cystic lesions in the brain [1]. This device helps to drain the cerebrospinal fluid and contents of cystic lesions. Additionally, intraventricular administration of some drugs, such as amphotericin B, primethamine and methotrexate, can be conducted through this device $[1,2]$. Although the usefulness of this device for treating cystic lesions in the brain has been previously reported, few reports of cystic brain metastases from lung cancer being controlled by the insertion of an Ommaya reservoir have been published in the English literature [3, 4]. In this report, we present a case of non-small-cell lung cancer (NSCLC) with a large cystic brain metastasis that was successfully controlled with the insertion of an Ommaya reservoir.

\section{Case report}

A 68-year-old male ex-smoker was referred to our hospital due to hoarseness and anarthria. Computed tomography (CT) revealed an irregular nodular shadow in the left upper lobe of the lung with enlargement of the left hilar, paraaortic and left subclavicular lymph nodes, which showed abnormal uptake of F-18 fluorodeoxyglucose on positron emission tomography/computed tomography (Fig. 1a). Magnetic resonance imaging (MRI) revealed a large mass in the left hemisphere (Fig. 1b, c) and multiple small nodules in the bilateral hemispheres (Fig. 1b, arrow). T1weighted images showed very low signal intensity and T2weighted images showed very high signal intensity in the largest mass consistent with the findings of a cystic lesion. 
Bronchoscopy was then performed and a direct biopsy revealed the tumor to be an adenocarcinoma. Based on these findings, the clinical stage was considered to be stage IV (cT1bN3M1b).

Due to the presence of multiple brain metastases with the one larger than $3 \mathrm{~cm}, 30$ Gray (Gy) of whole brain irradiation (WBI) was administered. Despite the administration of WBI, the largest cystic tumor continued to grow, and the surrounding edema expanded and the midline shifted (Fig. 1d). This resulted in worsened anarthria and the emergence of Gerstmann's syndrome. Various neurosurgical procedures were considered, and insertion of an Ommaya reservoir was chosen to minimize invasiveness. A dome-shaped plastic device was placed under the skin of the left head with a catheter positioned into the cavity of the cystic lesion without any complications (Fig. 2a). MRI and CT performed about 1 month after MRI as shown in Fig. 1c, revealed a reduction in the size of the tumor and the amount of surrounding edema (Fig. 2b, c). Crucially, the neurological symptoms improved. Since the cystic tumor was reduced from 4.7 to $2.9 \mathrm{~cm}, 20$ Gy of stereotactic radiosurgery (SRS) was administered. Although the cystic tumor remained the same size after the SRS was completed, no punctures were needed to drain the fluid. The patient is doing well at 6 months after the insertion of the Ommaya reservoir and is currently undergoing chemotherapy with carboplatin and pemetrexed due to the improvement of performance status (PS) from 3 to 1 .

\section{Discussion}

Ommaya reservoirs were originally developed to achieve aseptic access to ventricular cerebrospinal fluid. The device consists of an indwelling subcutaneous capsule made of silicone rubber that fits into a cranial burr-hole and is connected to a ventricular catheter. Percutaneous needle punctures can be repeatedly made through the dome of the capsule. This device is used for the administration of chemotherapy for neoplasia, cystic tumor drainage, ventricular drainage, special diagnostic studies and sampling of cerebrospinal fluid [1,2]. In addition, diverse types of diseases, such as primary brain tumors, fungal meningitis, toxoplasmosis and head injury, can be indications for the placement of the device [1]. However, few reports of cystic brain metastasis from lung cancer being controlled with the
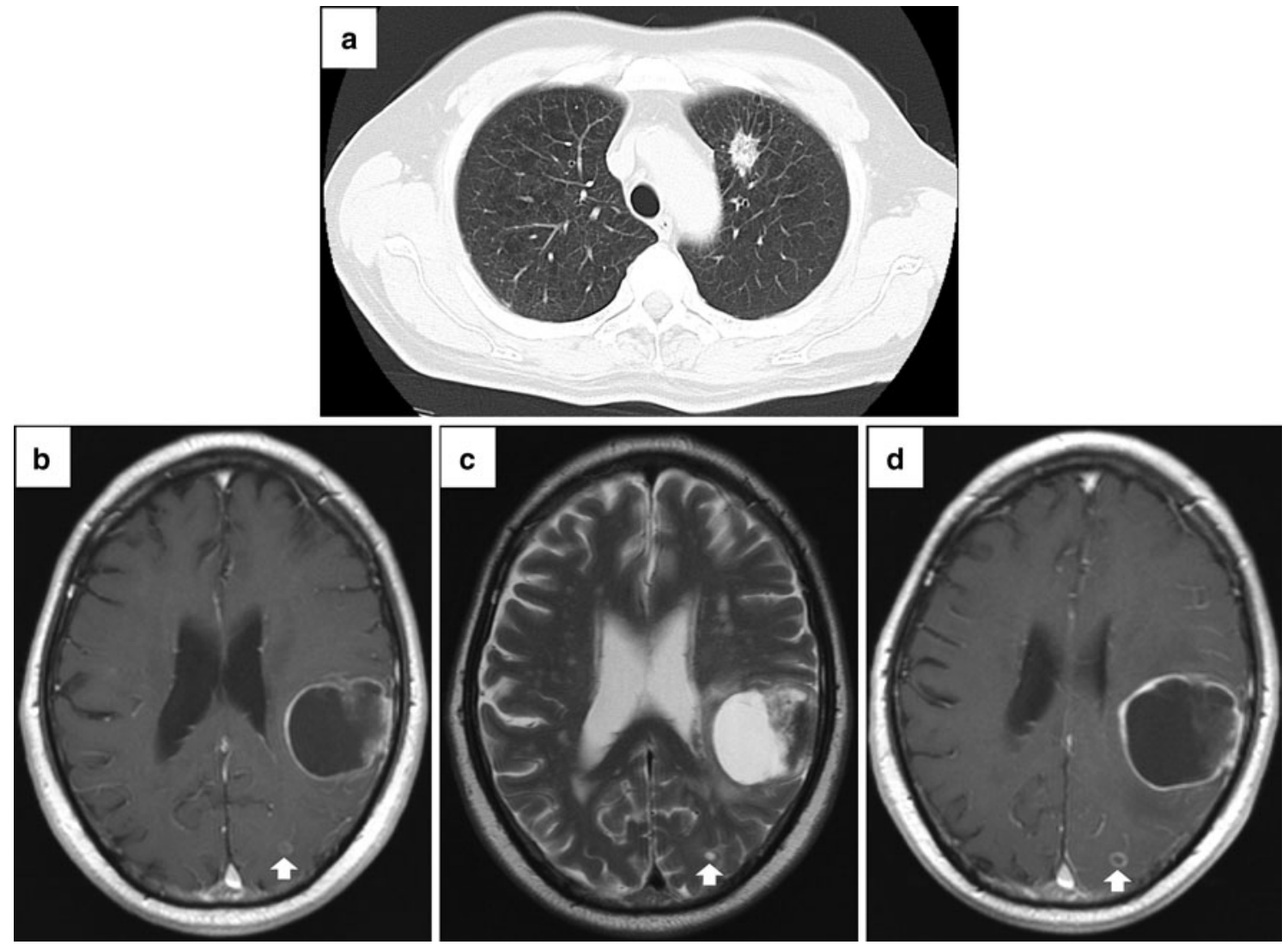

Fig. 1 CT and MRI findings. CT shows an irregular nodule in the left upper lobe of the lung (a). T1- and T2-weighted images indicate a before the administration of WBI (b, c). T1-weighted images show the tumor after WBI (d) 
Fig. 2 Ommaya reservoir placement. Left lateral and frontal views of the head show the Ommaya reservoir (asterisk) placed under the skin of the left head with a catheter positioned into the cavity of the cystic lesion (arrow) (a). MRI and CT images show the inserted Ommaya reservoir (b, asterisk, c)
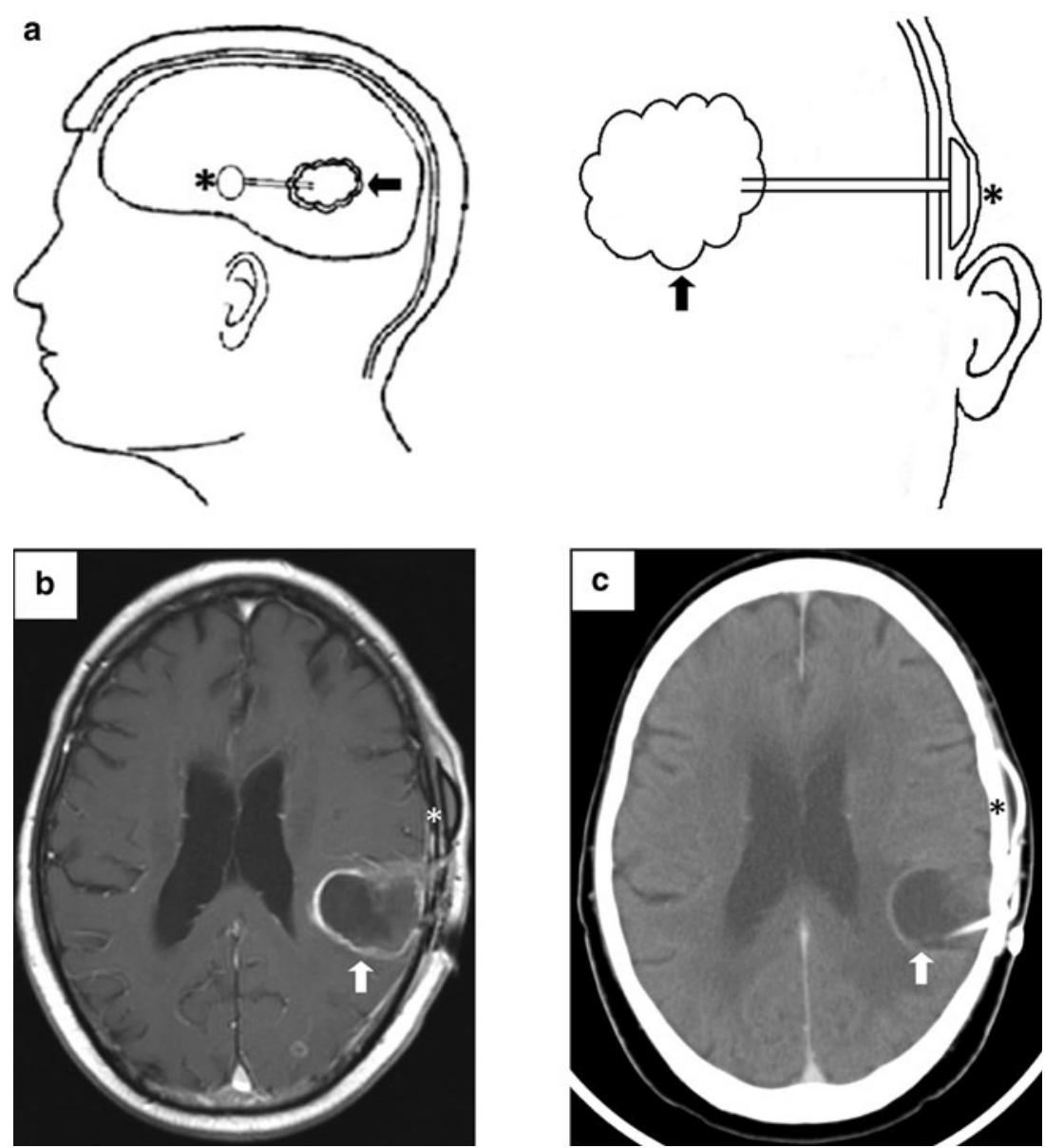

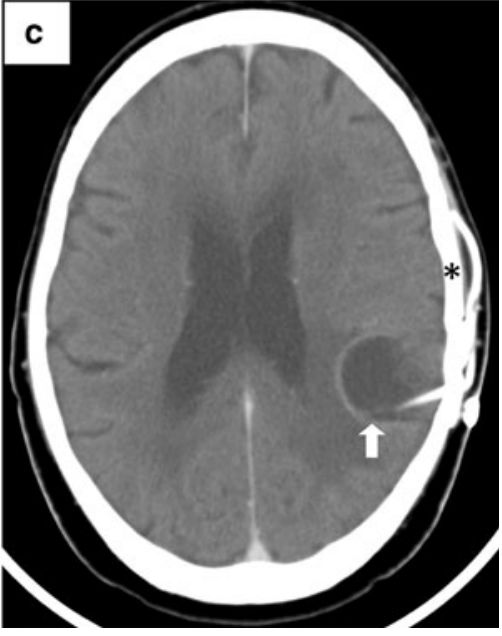

insertion of an Ommaya reservoir have been published in the English literature [3, 4]. Takeda and colleagues reported the case of a SCLC patient with solitary cystic brain metastasis who was successfully treated with a stereotactically inserted Ommaya reservoir followed by neurosurgical resection of the tumor, and the survival time of the patient after the insertion of the device was 4 months [3]. In addition, the usefulness of SRS following Ommaya reservoir placement has been reported in patients with large cystic metastatic brain tumors, including lung and breast cancer, with a median survival time of 7 months after the insertion of the device [4]. However, these papers did not mention chemotherapy after an Ommaya reservoir was placed. In the present case, a metastasized cystic lesion of the brain was controlled with the insertion of an Ommaya reservoir followed by the administration of stereotactic radiosurgery and chemotherapy, and the patient is currently doing well at 6 months, in comparison to the findings reported by others [3, 4], after the insertion of the device. Chemotherapy, as well as the insertion of the device, was also thought to have contributed the survival time of the patient, because the primary lesions and the metastasized lymph nodes were reduced after the administration of four cycles of carboplatin and pemetrexed.

Brain metastases are common in lung cancer patients, with an incidence of approximately $40 \%$. The majority of brain metastases of NSCLC are solid, whereas cystic brain metastases are exceedingly rare. While solid brain metastases are treated with optimal treatment modalities such as WBI, SRS or neurological surgery depending on the size and number of the metastases and the general conditions of the patient, cystic counterparts are generally resistant to radiation therapy, and neurosurgery of cystic lesions has been reported to be preferable to SRS or stereotactic aspiration when possible [5]. However, in the present case, due to the presence of multiple metastatic lesions, WBI was first chosen followed by insertion of an Ommaya reservoir for less invasiveness and to achieve ventricular drainage and drainage of the primary brain cystic tumors [1]. It is of note that no punctures were needed to drain the cystic tumors, as several punctures are generally required since the effusion typically fills the mass [3]. This may have been due to the reduction of viable cancer cells in the cystic wall by the multimodal treatments. Additionally, 
although few complications are associated with the placement of the device, attention should be paid to potential adverse events caused by chemotherapy, including leucopenia, neutropenia and thrombocytopenia by chemotherapy, which can lead to critical complications such as intracranial hemorrhage or infection.

In conclusion, the insertion of an Ommaya reservoir is therefore considered to be a useful treatment modality to improve the neurological symptoms and PS in NSCLC patients with cystic brain metastasis with minimal invasiveness, thus allowing for the administration of further neurosurgical therapy and chemotherapy which are crucial for the successful treatment of advanced NSCLC.

Conflict of interest No authors have any conflict of interest to disclose.

\section{References}

1. Ratcheson RA, Ommaya AK (1968) Experience with the subcutaneous cerebrospinal-fluid reservoir. Preliminary report of 60 cases. N Engl J Med 279:1025-1031

2. Witorsch P, Williams TW Jr, Ommaya AK et al (1965) Intraventricular administration of amphotericin B. Use of subcutaneous reservoir in four patients with mycotic meningitis. JAMA 194: 699-702

3. Takeda T, Saitoh M, Takeda S (2009) Solitary cystic brain metastasis of small-cell lung carcinoma controlled by a stereotactically inserted Ommaya reservoir. Am J Med Sci 337:215-217

4. Yamanaka Y, Shuto T, Kato Y et al (2006) Ommaya reservoir placement followed by Gamma Knife surgery for large cystic metastatic brain tumors. J Neurosurg 105:79-81

5. Kim MS, Lee SI, Sim SH (1999) Brain tumors with cysts treated with Gamma Knife radiosurgery: is microsurgery indicated? Stereotact Funct Neurosurg 72:38-44 